\title{
LEVANTAMENTO FITOSSOCIOLÓGICO DE DOIS FRAGMENTOS DA FLORESTA OMBRÓFILA MISTA, EM TIJUCAS DO SUL, PR
}

\section{Phitosociological Survey of Ttwo Fragments of Ombrophylous Mixed Forest, at Tijucas do Sul, PR.}

\author{
Sílvia Elaine Geraldi ${ }^{1}$ \\ Alexandre Bernardi Koehler ${ }^{2}$ \\ Érico Emed Kauano ${ }^{3}$
}

\section{Resumo}

Este trabalho objetivou caracterizar a composição florística e a estrutura de dois fragmentos da Floresta Ombrófila Mista, em Tijucas do Sul, PR. Para a tomada de dados foram medidas 10 parcelas temporárias (10 x $20 \mathrm{~m}$ ) em cada remanescente, incluindo todas as árvores com DAP $\geq 3,18 \mathrm{~cm}$. Foram registradas ao todo 88 espécies pertencentes a 61 gêneros e a 32 famílias. Em termos médios, para os dois ambientes, determinouse uma densidade de 2.620 indivíduos/ha. Na área A, a Cupania vernalis Cambess apresentou a maior densidade (320 árvores/ha), com uma freqüência de 90\%. Na área B, a principal espécie relacionada à densidade é a Nectandra megapotamica (Spreng.) Mez. (205 árvores/ha), sendo também a mais freqüente (90\%). A espécie dominante e mais importante, de acordo com o valor de importância, em ambas as áreas, é a Araucaria angustifolia (Bertol.) Kuntze. Dada a escassez de trechos de floresta nativa bem preservados na região, sugere-se a preservação irrestrita destes fragmentos, a fim de garantir a perpetuação da base genética que abrigam.

Palavras-chaves: Fitossociologia; Floresta Ombrófila Mista; Fragmento florestal.

\section{Abstract}

This work aimed the description of the floristic composition and the structure of two fragments of Ombrophylous Mixed Forest, in Tijucas do Sul, PR. For data collection were measured 10 temporary plots $(10 \times 20 \mathrm{~m})$ on each remainder, including all the tress with $\mathrm{DBH} \geq 3,18 \mathrm{~cm}$. There were registered 88 species, of 61 genus and 32 families. On average it was estimated a density of 2.620 trees/hectare. On area A, Cupania vernalis Cambess had the higher density (320 trees/hectare), and a frequency of $90 \%$. On area B, the main species according to density is Nectandra megapotamica (Spreng) Nees (205 trees/hectare), as well the most frequent (90\%). The dominant and most important species, according with the importance value, on both areas, is Araucaria angustifolia (Bertol.) Kuntze. In function of the scarcity of forest patches in the region it is suggested the unrestricted preservation of theses fragments in order to perpetuate the genetic basis sheltered by them.

Keywords Phytosociology; O mbrophylous Mixed Forest; forest fragment.

Bióloga, Instituto de Ecoturismo do Paraná. sunbio@ibest.com.br

2 Engenheiro Florestal, M.Sc., Pontifícia Universidade Católica do Paraná. alexandre.koehler@pucpr.br

3 Biólogo, Pontifícia Universidade Católica do Paraná. erico.kauano@hotmail.com 


\section{Introdução}

A vegetação da região sul do Brasil é bastante diversificada em termos fisionômicos, estruturais e florísticos. Neste mosaico destaca-se a Floresta Ombrófila Mista, mais conhecida como Floresta com Araucária ou Pinheiral. Segundo Klein (1979a), este tipo de floresta se estendia, especialmente, por parte do Planalto Meridional, de São Paulo até o norte do Rio Grande do Sul, recobrindo contínuas áreas, nos estados do Paraná e Santa Catarina. Nos estados do Rio de Janeiro e Minas Gerais esta formação florestal ocorria isoladamente e uma pequena área penetrava, também, a Argentina (HUECK, 1972). O termo Mista, empregado para sua designação fitogeográfica, provém da mistura de floras de diferentes origens, com gêneros australásicos (Drimys e Araucaria) e afro-asiáticos (Podocarpus) (IBGE, 1992).

No Paraná, segundo Maack (1968), a região da Floresta Ombrófila Mista iniciava no primeiro planalto, imediatamente a oeste da Serra do Mar, estendendo-se também pelo segundo e terceiro planaltos do estado, com uma superfície original correspondendo a 37\% do ternitório e os ecossistemas a ela associados, campos naturais, cerrados, matas de galeria e várzeas, perfazendo mais $16 \%$ da superfície paranaense.

Infelizmente, espécies típicas da formação, como a araucária (Araucaria angu stifolia), a imbuia (Ocotea porosa), o sassafrás (Ocotea odorifera) e o xaxim-bugio (Dicksonia sellowiana), em face de suas características e valor econômico elevado, encontram-se hoje desfavorecidas, não só pela intervenção destruidora do homem, mas pe- las modificações daí geradas. Essas espécies figuram na lista oficial de espécies ameaçadas de extinção, entre outros fatores, devido à vulnerabilidade a qual estão sujeitas as suas populações naturais em função da grande pressão exercida sobre os últimos remanescentes (SEMA/GTZ, 1995).

Em função da exploração desenfreada muito pouco restou da Floresta Ombrófila Mista no Paraná. Segundo o Ministério do Meio Ambiente (2004), não existem mais remanescentes primários e os secundários representam apenas 0,8\% da área do estado, em estágio avançado de sucessão, e 22,3\% em estágio inicial e médio.

Este trabalho visa a contribuir na conservação dos remanescentes da Floresta Ombrófila Mista, por meio do levantamento da estrutura e composição florística de dois trechos deste tipo de floresta. Estas informações poderão servir como auxílio na elaboração de planos de recomposição florestal na região de estudo.

\section{Material e Métodos}

Os dois remanescentes localizam-se no município de Tijucas do Sul, Região Metropolitana de Curitiba, no estado do Paraná, a cerca de 70 $\mathrm{km}$ da capital do estado (FIGURA 1). A altitude média encontra-se na faixa dos 850 m s.n.m., sobre o Primeiro Planalto Paranaense. 0 clima predominante da região, conforme a classificação de Köppen, é do tipo Cfb, sendo definido como subtropical úmido, com ocorrência de geadas entre os meses de junho e agosto. 


\section{Figura 1 - Localização das áreas de estudo.}

Figure 1 - Location of the study areas.

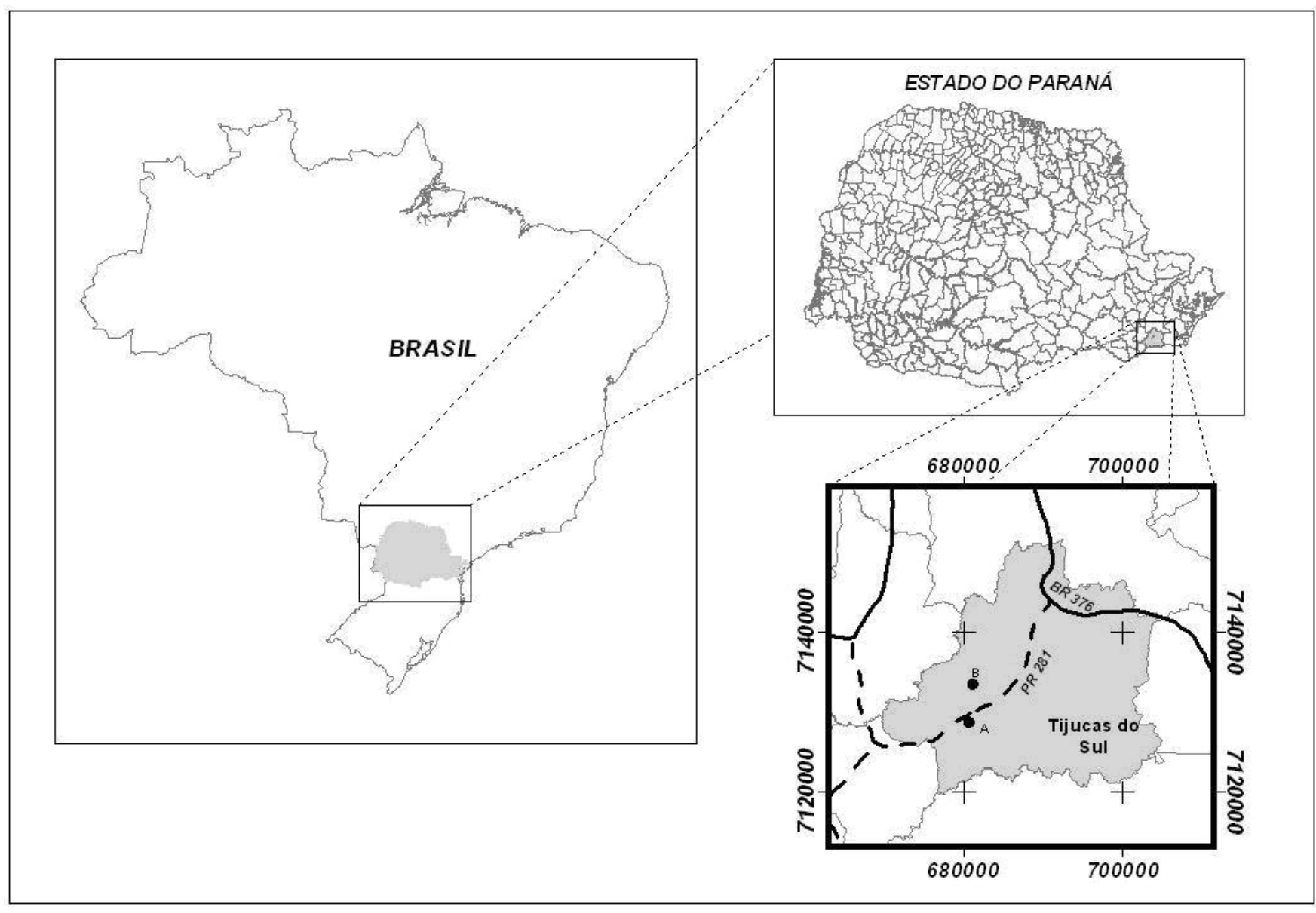

O primeiro remanescente estudado (área A) pertence à Associação Paranaense de Cultura, possui cerca de 6 ha e faz parte da área do Vivat Floresta Sistemas Ecológicos, um projeto conservacionista conduzido pela Instituição Filantrópica Sergius Endelyi em parceria com a Pontifícia Universidade Católica do Paraná. O segundo remanescente (área B), de propriedade do Senhor Beno Bührer, possui uma área de 30 ha e localiza-se nas cercanias do centro urbanizado do município.

Para a realização do levantamento estrutural foram demarcadas vinte unidades amostrais retangulares de duzentos metros quadrados cada (10 x 20 m), sendo dez em cada trecho. A locação das unidades amostrais foi totalmente aleatória, procurando-se evitar as porções mais externas dos fragmentos a fim de se eliminar a influência do efeito de borda.

Foram consideradas todas as árvores com o DAP maior do que 3,14 centímetros e de cada uma anotou-se o nome comum, a circunferência, a altura do peito (CAP) e a altura total estimada. $\mathrm{O}$ material botânico coletado foi identificado no HUCP, o Herbário do Centro de Ciências Biológicas e de Saúde da PUCPR, e o material vegetativo foi aproveitado em um herbário de campo. As exsicatas da família Myrtaceae foram determinadas pelo botânico Marcos Sobral da UFMG e, as demais, pelos autores deste trabalho.

\section{Resultados e discussão}

Nas vinte parcelas estudadas foram mensurados um total de 1.051 indivíduos ( $\mathrm{DAP} \geq 3,14$ $\mathrm{cm})$, dos quais 72 estavam mortos, porém ainda em pé. Foram registradas 88 espécies, pertencentes a 61 gêneros e 34 famílias. Do número total de árvores mensuradas, a área A apresentou 539 indivíduos, pertencentes a 54 espécies, 43 gêneros e 
25 famílias. Na área B os 512 indivíduos amostrados dividem-se em 66 espécies, 51 gêneros e 29 famílias. Do total de espécies 32 são comuns às duas áreas, perfazendo 36,3\% da composição florística total (TABELA 1).

As famílias mais representadas nas comunidades estudadas são Myrtaceae (21 spp.), Lauraceae (9 spp.), Asteraceae (7spp.), Rubiaceae (6 spp.) e Aquifoliaceae (4 spp.), totalizando 27 gêneros e 47 espécies.

A diversidade de espécies arbóreas da família Myrtaceae é expressiva em ambas as áreas e corrobora com os trabalhos de Jarenkow (1985), Galvão et al. (1989), Nascimento et al. (2001) e Rondon Neto et al. (2002), os quais afirmam que as comunidades nos domínios da Floresta Ombrófila Mista constituem um importante centro de concentração de espécies desta família.

As principais espécies da área A, em número de indivíduos por hectare, são Cupania vernalis (320 árvores/ha), Drimys brasiliensis (295 árvores/ha), Casearia decandra (235 árvores/ha), Myrsine umbellata (205 árvores/ha) e juntas elas representam cerca de $40 \%$ de todas as árvores da comunidade (TABELA 2). Na área B (TABELA 3), as principais espécies, em relação à densidade absoluta, são Nectandra megapotamica (205 árvores/ha), Cupania vernalis (185 árvores/ha), Alibertia concolor (180 árvores/ha) e a Ocotea odorifera (120 árvores/ha), que juntas acumulam 30\% da densidade relativa.

As espécies dominantes, na área $\mathrm{A}$, são Araucaria angustifólia, com 5,79 m_/há; Ocotea porosa, com 3,05 m_/há; Casearia decandra, com 1,54 m_/há; Campomanesia xanthocarpa, com 1,08 m_/ há; Drimys brasiliensis, com 1,06 m_/ ha e Cupania vernalis, com 1,05 m_/há; Podocarpus lambertii, com 0,96 m/ha e Nectandra megapotamica, com 0,88 $\mathrm{m} /$ /ha. Juntas, essas perfazem $61,50 \%$ da dominância relativa total. Na área B, as espécies dominantes são Araucaria angustifólia, com 14,22 m_/ há; Nectandra megapotamica, com
4,92 m / há; Gordonia fruticosa, com 2,64 m /há; Ilex paraguariensis, com 2,37 m_/há; Drimys brasiliensis, com 1,35 m / há; Alibertia concolor, com 1,19 m_/há; Pimenta pseudocaryophyllus, com 1,17 $\mathrm{m}$ / ha e Prunus sellowii, com 1,02 m_/ ha. O montante da dominância relativa, representado por estas espécies, encerra $63,60 \%$.

Dentre as mais freqüentes na área A merecem destaque as espécies Cupania vernalis e Drimys brasiliensis. Essas são as mais representativas, estando presentes em $90 \%$ das unidades amostrais deste local. Casearia decandra, Myrsine umbellata, Podocarpus lambertii, Aegiphila sellowiana, Pimenta pseudocaryophyllus e Myrcia fallax surgem logo em seguida com 80\% de presença nas unidades amostrais (TABELA 2). Na área B as espécies Nectandra megapotamica e Alibertia concolor estão presentes em $90 \%$ do total de parcelas estabelecidas. As outras espécies que apresentam maiores freqüências absolutas são Araucaria angustifolia e Ocotea odorífera, sendo cada uma representada em $80 \%$ das unidades amostrais, Ilex paraguariensis e Cupania vernalis em $70 \%$ cada uma. Juntas perfazem $22,44 \%$ da freqüência relativa (TABELA 3).

Na área A (TABELA 2), a Araucaria angustifolia é a espécie mais importante de acordo com valor de importância, assim como na área $B$ (TABELA 3), embora nesta a espécie assuma um valor de importância maior em relação à comunidade. É evidente e expressiva a importância que a Araucaria angustifolia apresenta nas áreas, não só como tipificadora da fisionomia das comunidades, mas também como indicador do grau de conservação dos fragmentos. Resultado semelhante foi obtido por Longhi (1980), em que a Araucaria angustifolia apresentou uma importância muito superior às demais espécies, o equivalente a 10\% do valor total. Na Reserva Genética de Caçador, SC, segundo Silva et al. (1993), o valor de importância da Araucaria angustifolia correspondeu a $30 \%$, aproximadamente, em relação ao total da comunidade. 
Tabela 1 - Lista de espécies encontradas nas áreas A e B, ordenadas por família. Table 1 - Species list encountered on areas A and B, positioned by family.

\begin{tabular}{|c|c|c|c|c|c|}
\hline ESPÉCIES POR FAMÍLIA & ÁREA A & ÁREA B & ESPÉCIES POR FAMÍLIA & ÁREA A & ÁREA B \\
\hline Anacardiaceae & & & 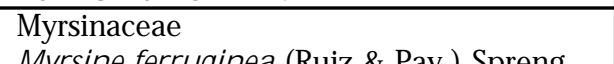 & & \\
\hline Lithraea brasiliensis Marchand & $\mathrm{X}$ & & Myrsine ferruginea (Ruiz \& Pav.) Spreng. & & $\begin{array}{l}\mathrm{X} \\
\mathrm{X}\end{array}$ \\
\hline aceae & & & Myrsine umbellata Mart. & $\mathrm{X}$ & $\mathrm{X}$ \\
\hline Rollinia rugulosa Schltdl. & $\mathrm{X}$ & $\mathrm{X}$ & Myrtaceae & & \\
\hline Aquifoliaceae & & & Blepharocalyx salicifolius (Kunth) O. Berg & $\mathrm{X}$ & $\mathrm{X}$ \\
\hline Ilex dumosa Reissek & $\mathrm{X}$ & $\mathrm{X}$ & Campomanesia xanthocarpa (Mart.) O. Berg & $\mathrm{X}$ & $\mathrm{X}$ \\
\hline Ilex paraguariensis A. St.-Hil. & & $\begin{array}{l}\Lambda \\
\mathrm{X}\end{array}$ & Calyptranthes grandifolia O. Berg & $\mathrm{X}$ & $\mathrm{X}$ \\
\hline Ilex theezans Mart. & $\mathrm{X}$ & X & Eugenia cereja D. Legrand & & $\mathrm{X}$ \\
\hline Ilex sp. & & $\mathrm{X}$ & Eugenia neoverrucosa Sobral & $\mathrm{X}$ & $\mathrm{X}$ \\
\hline Araucariaceae & & & latysema O. Berg. & $\mathrm{X}$ & \\
\hline Araucaria angustifolia (Bertol.) Kuntze & $\mathrm{X}$ & $\mathrm{X}$ & $\begin{array}{l}\text { Eugenia sp. } \\
\text { Gomidesia affinis (Cambess.) Leor. }\end{array}$ & $\mathrm{X}$ & $\mathrm{X}$ \\
\hline Ast & & & Myrceugenia c.f. venosa & & $\begin{array}{l}\lambda \\
\mathrm{X}\end{array}$ \\
\hline Asteraceae 1 & $\mathrm{x}$ & $X$ & Myrceugenia ovata (Hook. Et Arn.) O. Berg. & & $\mathrm{X}$ \\
\hline $\begin{array}{l}\text { Piptocarpha angustifolia Dusén ex Malme } \\
\text { Piptocarpha axillaris Baker. }\end{array}$ & $\begin{array}{l}X \\
X\end{array}$ & & Myrcia fallax (Rich.) DC. & $\mathrm{X}$ & $\mathrm{X}$ \\
\hline Piptocarpha sp. & & $\mathrm{X}$ & Myrcia hatschbachii D. Lebrand & & $\mathrm{X}$ \\
\hline Gochnatia polymorpha (Less.) Cabrera & $\mathrm{X}$ & $\mathrm{X}$ & $\begin{array}{l}\text { ustris DC. } \\
\text { nardiana (O. Berq) Kiaersk. }\end{array}$ & $X$ & $X$ \\
\hline color (Spreng.) Less. & $\mathrm{X}$ & $\mathrm{X}$ & Myrcia selloi (Spreng.) N. Silveira & $\mathrm{X}$ & $\begin{array}{l}\lambda \\
X\end{array}$ \\
\hline pus c.f. lymansmithii & $\mathrm{X}$ & & Myrcia sp.1 & $\mathrm{X}$ & $\mathrm{X}$ \\
\hline Big & & & Myrtaceae 1 & $\mathrm{X}$ & $\mathrm{X}$ \\
\hline micrantha Cham. & $\mathrm{X}$ & & Myrtaceae 2 & $\mathrm{X}$ & \\
\hline $\begin{array}{l}\text { Canellaceae } \\
\text { Capsicodendron dinisii (Schwacke) Occhioni }\end{array}$ & $\mathrm{X}$ & $\mathrm{X}$ & Myrtaceae 3 & $\mathrm{x}$ & $X$ \\
\hline Clethraceae & & & Pimenta pseudocaryophyllus (Gomes) Landrum & $\mathrm{X}$ & $\begin{array}{l}\Lambda \\
X\end{array}$ \\
\hline abra Pers. & & $\mathrm{X}$ & Podocarpaceae & & \\
\hline 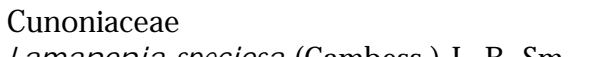 & & & Podocarpus lambertii Klotzsch ex Eichler & $\mathrm{X}$ & $\mathrm{X}$ \\
\hline $\begin{array}{l}\text { Lamanonia speciosa (Cambess.) L. B. Sm. } \\
\text { Elaeocarpaceae }\end{array}$ & $\mathrm{X}$ & $\mathrm{X}$ & $\begin{array}{l}\text { Proteaceae } \\
\text { Roupala brasiliensis Klotzsch }\end{array}$ & & $\mathrm{X}$ \\
\hline Sloanea & & $\mathrm{X}$ & Rhamnaceae & & \\
\hline $\begin{array}{l}\text { eae } \\
\text { triplinervia (Spreng.) }\end{array}$ & & $X$ & Hovenia dulcis Thunb. & $\mathrm{X}$ & \\
\hline $\begin{array}{l}\text { triplinervia (Spreng.) } \\
\text { andulatum (Vel.) Pax }\end{array}$ & $\mathrm{X}$ & $\mathrm{X}$ & $\begin{array}{l}\text { Rosaceae } \\
\text { Prunus sellowii Koehne }\end{array}$ & $\mathrm{X}$ & $\mathrm{X}$ \\
\hline Sebastiana commersoniana (Baill) Smith \& Downs & $\mathrm{X}$ & & Rubiaceae & & \\
\hline Fabaceae & & & Alibertia concolor (Cham.) K. Schum. & & $\begin{array}{l}\mathrm{X} \\
\mathrm{X}\end{array}$ \\
\hline Machaerium c.f. paraguariensis & & $\mathrm{X}$ & Coussarea sp. & & $\begin{array}{l}X \\
X\end{array}$ \\
\hline $\begin{array}{l}\text { Machaerium stiptatum (DC.) Vogel } \\
\text { Machaerium sp. }\end{array}$ & $\begin{array}{l}\mathrm{X} \\
\mathrm{X}\end{array}$ & & $\begin{array}{l}\text { Psychotria suterella Müll. Arg. } \\
\text { Psychotria sp. } 1\end{array}$ & $\mathrm{X}$ & $\begin{array}{l}X \\
X\end{array}$ \\
\hline $\begin{array}{l}\text { Ma } \\
\text { Fla }\end{array}$ & $X$ & & Rubiaceae 1 & & $\mathrm{X}$ \\
\hline ndra Jacq. & $\mathrm{X}$ & $\mathrm{X}$ & Rubiaceae 2 & & $\mathrm{X}$ \\
\hline ris Sw. & $\mathrm{X}$ & & Sapindaceae & & \\
\hline Casearia obliqua Spreng. & $\mathrm{X}$ & & $\begin{array}{l}\text { Cupania vernalis Cambess. } \\
\text { Matayba elaegnoides Radlk }\end{array}$ & $\begin{array}{l}\mathrm{X} \\
\mathrm{X}\end{array}$ & $\begin{array}{l}X \\
X\end{array}$ \\
\hline vesiculosum (Nees) Kosterm. & & $\mathrm{X}$ & Simaroubaceae & & \\
\hline 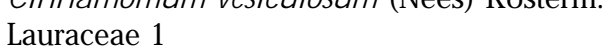 & $\mathrm{X}$ & & Picramnia parvifolia Engl. & $\mathrm{X}$ & $\mathrm{X}$ \\
\hline a megapotamica (Spreng.) Mez. & $\mathrm{X}$ & $\mathrm{X}$ & $\begin{array}{l}\text { Solanaceae } \\
\text { Solanum sn } 1\end{array}$ & & \\
\hline Nectandra c.f. lanceolata & $\mathrm{X}$ & $\mathrm{X}$ & Solan & $\mathrm{X}$ & $\mathrm{X}$ \\
\hline Occ & & $\mathrm{v}$ & Sorm & & \\
\hline Ocotea odorifera (Vellozo) Rohwer & $\mathrm{X}$ & 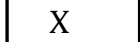 & Symplocaceae & & $\mathrm{X}$ \\
\hline Ocotea porosa (Nees \& C. Mart.) Barroso & $\mathrm{X}$ & & symplocos unillord & & \\
\hline Ocotea puberula (Rich.) Ness & $\mathrm{X}$ & $\mathrm{X}$ & Gordonia fruticosa (Schrad.) H. Keng & & $\mathrm{X}$ \\
\hline $\begin{array}{l}\text { Ocotea sp. } \\
\text { Melastomataceae }\end{array}$ & & $X$ & Ulmaceae & & \\
\hline Tibouchina sellowiana Cogn. & & $\mathrm{X}$ & $\begin{array}{l}\text { Trema micrantha (L.) Blume } \\
\text { Verhenaceae }\end{array}$ & & \\
\hline Tibouchina sp. & $\mathrm{X}$ & & Aegiphila sellowiana Cham. & & $\mathrm{X}$ \\
\hline $\begin{array}{l}\mathrm{Me} \\
\mathrm{Ce}\end{array}$ & $\mathrm{Y}$ & & Vitex megapotamica (Spreng.) Moldenke & $\mathrm{x}$ & \\
\hline $\begin{array}{l}\text { Cedrela fissills } \\
\text { Moraceae }\end{array}$ & $X$ & & $\begin{array}{l}\text { Winteraceae } \\
\text { Drimvs brasilie }\end{array}$ & $\mathrm{X}$ & $\mathrm{X}$ \\
\hline & & $\mathrm{X}$ & & & $\mathrm{X}$ \\
\hline Soracea bonplandii (Baillon) W. Burger & & $\mathrm{X}$ & N.i. 2 & $\mathrm{X}$ & \\
\hline
\end{tabular}


Tabela 2 - Fitossociologia da comunidade arbórea da área A. Sendo: DA - Densidade Absoluta; DR - Densidade Relativa; DoA - Dominância Absoluta; DoR - Dominância Relativa; FA Freqüência Absoluta; FR - Freqüência Relativa; VI - Valor de Importância.

Table 2 - Phitosociology of tree community on area B. DA - Absolute Density; DR - Relative Density; DoA - Absolute dominancy; DoR - Relative Dominance; FA - Absolute Frequency; FR -Relative Frequency; VI - Importanœe Value.

\begin{tabular}{|c|c|c|c|c|c|c|c|}
\hline \multirow[t]{2}{*}{ Nome Científico } & \multicolumn{2}{|c|}{ Densidade } & \multicolumn{2}{|c|}{ Dominância } & \multicolumn{2}{|c|}{ Frequência } & \multirow[t]{2}{*}{ VI } \\
\hline & $\begin{array}{l}\text { DA } \\
\text { (n/ha) }\end{array}$ & $\begin{array}{l}\text { DR } \\
\text { (\%) }\end{array}$ & $\begin{array}{l}\text { DoA } \\
\left(\mathrm{m}^{2} / \mathrm{ha}\right)\end{array}$ & $\begin{array}{l}\text { DoR } \\
\text { (\%) }\end{array}$ & $\begin{array}{l}\text { FA } \\
\text { (\%) }\end{array}$ & $\begin{array}{l}\text { FR } \\
\text { (\%) }\end{array}$ & \\
\hline Araucaria angustifolia & 60 & 2,22 & 5,7922 & 23,07 & 50 & 2,81 & 28,11 \\
\hline Cupania vernalis & 320 & 11,85 & 1,0505 & 4,18 & 90 & 5,06 & 21,09 \\
\hline Drimys brasiliensis & 295 & 10,93 & 1,0642 & 4,24 & 90 & 5,06 & 20,22 \\
\hline Casearia decandra & 235 & 8,70 & 1,5428 & 6,15 & 80 & 4,49 & 19,34 \\
\hline Ocotea porosa & 85 & 3,15 & 3,0551 & 12,17 & 60 & 3,37 & 18,69 \\
\hline Myrsine umbellata & 205 & 7,59 & 0,7949 & 3,17 & 80 & 4,49 & 15,25 \\
\hline Podocarpus lambertii & 120 & 4,44 & 0,9609 & 3,83 & 80 & 4,49 & 12,77 \\
\hline Aegiphila sellowiana & 90 & 3,33 & 0,5979 & 2,38 & 80 & 4,49 & 10,21 \\
\hline Campomanesia xanthocarpa & 45 & 1,67 & 1,0841 & 4,32 & 60 & 3,37 & 9,36 \\
\hline Pimenta pseudocaryophyllus & 60 & 2,22 & 0,5615 & 2,24 & 80 & 4,49 & 8,95 \\
\hline Myrcia fallax & 80 & 2,96 & 0,3546 & 1,41 & 80 & 4,49 & 8,87 \\
\hline Nectandra megapotamica & 55 & 2,04 & 0,8876 & 3,54 & 40 & 2,25 & 7,82 \\
\hline Casearia sylvestris & 95 & 3,52 & 0,3031 & 1,21 & 50 & 2,81 & 7,53 \\
\hline Casearia obliqua & 85 & 3,15 & 0,3325 & 1,32 & 50 & 2,81 & 7,28 \\
\hline Blepharocalyx salicifolius & 50 & 1,85 & 0,4995 & 1,99 & 50 & 2,81 & 6,65 \\
\hline Psychotria sp. 1 & 75 & 2,78 & 0,3312 & 1,32 & 40 & 2,25 & 6,34 \\
\hline Eugenia neoverrucosa & 55 & 2,04 & 0,2717 & 1,08 & 20 & 1,12 & 4,24 \\
\hline Eugenia platysema & 40 & 1,48 & 0,1195 & 0,48 & 40 & 2,25 & 4,20 \\
\hline Piptocarpha angustifolia & 10 & 0,37 & 0,6533 & 2,60 & 20 & 1,12 & 4,10 \\
\hline Matayba elaegnoides & 35 & 1,30 & 0,0596 & 0,24 & 40 & 2,25 & 3,78 \\
\hline Ocotea odorifera & 40 & 1,48 & 0,2500 & 1,00 & 20 & 1,12 & 3,60 \\
\hline Ocotea puberula & 25 & 0,93 & 0,0839 & 0,33 & 40 & 2,25 & 3,51 \\
\hline Ilex theezans & 35 & 1,30 & 0,1220 & 0,49 & 30 & 1,69 & 3,47 \\
\hline Myrcia palustris & 30 & 1,11 & 0,1609 & 0,64 & 30 & 1,69 & 3,44 \\
\hline Prunus sellowi & 15 & 0,56 & 0,2137 & 0,85 & 30 & 1,69 & 3,09 \\
\hline Myrtaceae 2 & 20 & 0,74 & 0,1885 & 0,75 & 20 & 1,12 & 2,62 \\
\hline Simphyopappus c.f. lymansmithii & 20 & 0,74 & 0,1865 & 0,74 & 20 & 1,12 & 2,61 \\
\hline Myrcia sp.1 & 25 & 0,93 & 0,1191 & 0,47 & 20 & 1,12 & 2,52 \\
\hline Cedrela fissilis & 5 & 0,19 & 0,4221 & 1,68 & 10 & 0,56 & 2,43 \\
\hline Rollinia rugulosa & 15 & 0,56 & 0,0217 & 0,09 & 30 & 1,69 & 2,33 \\
\hline Vernonia discolor & 10 & 0,37 & 0,2349 & 0,94 & 10 & 0,56 & 1,87 \\
\hline Tibouchina sp. & 10 & 0,37 & 0,0491 & 0,20 & 20 & 1,12 & 1,69 \\
\hline Myrtaceae 1 & 25 & 0,93 & 0,0418 & 0,17 & 10 & 0,56 & 1,65 \\
\hline Sapium glandulatum & 5 & 0,19 & 0,2238 & 0,89 & 10 & 0,56 & 1,64 \\
\hline Sebastiana commersoniana & 20 & 0,74 & 0,0588 & 0,23 & 10 & 0,56 & 1,54 \\
\hline Jacaranda micrantha & 10 & 0,37 & 0,0758 & 0,30 & 10 & 0,56 & 1,23 \\
\hline Calyptranthes grandifolia & 5 & 0,19 & 0,0965 & 0,38 & 10 & 0,56 & 1,13 \\
\hline Ilex dumosa & 5 & 0,19 & 0,0955 & 0,38 & 10 & 0,56 & 1,13 \\
\hline Lamanonia speciosa & 10 & 0,37 & 0,0335 & 0,13 & 10 & 0,56 & 1,07 \\
\hline Vitex megapotamica & 5 & 0,19 & 0,0736 & 0,29 & 10 & 0,56 & 1,04 \\
\hline Lithraea brasiliensis & 5 & 0,19 & 0,0637 & 0,25 & 10 & 0,56 & 1,00 \\
\hline Nectandra c.f. lanceolata & 5 & 0,19 & 0,0637 & 0,25 & 10 & 0,56 & 1,00 \\
\hline Gochnatia polymorpha & 5 & 0,19 & 0,0229 & 0,09 & 10 & 0,56 & 0,84 \\
\hline Hovenia dulcis & 5 & 0,19 & 0,0210 & 0,08 & 10 & 0,56 & 0,83 \\
\hline Capsicodendron dinisii & 5 & 0,19 & 0,0193 & 0,08 & 10 & 0,56 & 0,82 \\
\hline N.i. 2 & 5 & 0,19 & 0,0182 & 0,07 & 10 & 0,56 & 0,82 \\
\hline Piptocarpha axillaris & 5 & 0,19 & 0,0159 & 0,06 & 10 & 0,56 & 0,81 \\
\hline Myrcia selloi & 5 & 0,19 & 0,0090 & 0,04 & 10 & 0,56 & 0,78 \\
\hline Machaerium sp. & 5 & 0,19 & 0,0078 & 0,03 & 10 & 0,56 & 0,78 \\
\hline Solanum sp.1 & 5 & 0,19 & 0,0078 & 0,03 & 10 & 0,56 & 0,78 \\
\hline Eugenia sp. & 5 & 0,19 & 0,0067 & 0,03 & 10 & 0,56 & 0,77 \\
\hline Myrtaceae 3 & 5 & 0,19 & 0,0057 & 0,02 & 10 & 0,56 & 0,77 \\
\hline Picramnia parvifolia & 5 & 0,19 & 0,0048 & 0,02 & 10 & 0,56 & 0,77 \\
\hline Machaerium stiptatum & 5 & 0,19 & 0,0048 & 0,02 & 10 & 0,56 & 0,77 \\
\hline Mortas & 200 & 7,41 & 1,7626 & 7,02 & 90 & 5,06 & 19,49 \\
\hline TOTAL & 2700 & 100,00 & 25,1020 & 100,00 & 1780 & 100,00 & 300,00 \\
\hline
\end{tabular}


Tabela 3 - Fitossociologia da comunidade arbórea da área B. Sendo: DA - densidade absoluta; DR densidade relativa; DoA - dominância absoluta; DoR - dominância relativa; FA -freqüência absoluta; FR freqüência relativa; VI - valor de importância.

Table 3 - Phitosociology of tree community on area B. DA - Absolute Density; DR - Relative Density; DoA- AbsoluteDominancy; DoR- Relative Dominanœe, FA- AbsoluteFrequency; FR- Relativefrequency; V- ImportanœVValue.

\begin{tabular}{|c|c|c|c|c|c|c|c|}
\hline \multirow[t]{2}{*}{ Nome Científico } & \multicolumn{2}{|c|}{ Densidade } & \multicolumn{2}{|c|}{ Dominância } & \multicolumn{2}{|c|}{ Frequência } & \multirow[t]{2}{*}{ VI } \\
\hline & $\begin{array}{l}\text { DA } \\
\text { (n/ha) }\end{array}$ & $\begin{array}{l}\text { DR } \\
\text { (\%) }\end{array}$ & $\begin{array}{l}\text { DoA } \\
\text { (m²/ha) }\end{array}$ & $\begin{array}{l}\text { DoR } \\
\text { (\%) }\end{array}$ & $\begin{array}{l}\text { FA } \\
\text { (\%) }\end{array}$ & $\begin{array}{l}\text { FR } \\
\text { (\%) }\end{array}$ & \\
\hline Araucaria angustifolia & 60 & 2,22 & 5,7922 & 23,07 & 50 & 2,81 & 28,11 \\
\hline Araucaria angustifolia & 105 & 4,13 & 14,2256 & 31,29 & 80 & 3,74 & 39,16 \\
\hline Nectandra meqapotamica & 205 & 8,06 & 4,9242 & 10,83 & 90 & 4,21 & 23,09 \\
\hline Alibertia concolor & 180 & 7,07 & 1,1945 & 263 & 90 & 4,21 & 13,91 \\
\hline Gordonia fruticosa & 110 & 4,32 & 2,6464 & 5,82 & 60 & 2,80 & 12,95 \\
\hline Ilex paraguariensis & 85 & 3,34 & 2,3704 & 5,21 & 70 & 3,27 & 11,83 \\
\hline Cupania vernalis & 185 & 7,27 & 0,5813 & 1,28 & 70 & 3,27 & 11,82 \\
\hline Ocotea odorifera & 120 & 4,72 & 0,8455 & 1,86 & 80 & 3,74 & 10,31 \\
\hline Drimys brasiliensis & 105 & 4,13 & 1,3554 & 2,98 & 60 & 2,80 & 9,91 \\
\hline Psychotria sp. 1 & 115 & 4,52 & 0,4637 & 1,02 & 60 & 2,80 & 8,34 \\
\hline Pimenta pseudocaryophyllus & 60 & 2,36 & 1,1752 & 2,59 & 60 & 2,80 & 7,75 \\
\hline Casearia decandra & 100 & 3,93 & 0,6361 & 1,40 & 50 & 2,34 & 7,66 \\
\hline Podocarpus lambertii & 55 & 2,16 & 0,9306 & 2,05 & 50 & 2,34 & 6,54 \\
\hline Myrceu genia c.f. venosa & 105 & 4,13 & 0,2447 & 0,54 & 40 & 1,87 & 6,53 \\
\hline Prunus sellowii & 45 & 1,77 & 1,0263 & 2,26 & 50 & 2,34 & 6,36 \\
\hline Myrcia hatschbachii & 50 & 1,96 & 0,6206 & 1,37 & 60 & 2,80 & 6,13 \\
\hline Myrcia fallax & 45 & 1,77 & 0,4242 & 0,93 & 60 & 2,80 & 5,51 \\
\hline Eugenia neoverrucosa & 50 & 1,96 & 0,3278 & 0,72 & 50 & 2,34 & 5,02 \\
\hline Clethra scabra & 30 & 1,18 & 0,9588 & 2,11 & 30 & 1,40 & 4,69 \\
\hline Matayba ela egnoides & 40 & 1,57 & 0,2729 & 0,60 & 50 & 2,34 & 4,51 \\
\hline Myrsine umbellata & 35 & 1,38 & 0,263 & 0,58 & 40 & 1,87 & 3,82 \\
\hline Myrcia sp.1 & 45 & 1,77 & 0,1446 & 0,32 & 30 & 1,40 & 3,49 \\
\hline Ocotea puberula & 15 & 0,59 & 0,5665 & 1,25 & 30 & 1,40 & 3,24 \\
\hline Myrcia richardiana & 30 & 1,18 & 0,2634 & 0,58 & 30 & 1,40 & 3,16 \\
\hline Ilex dumosa & 15 & 0,59 & 0,503 & 1,11 & 30 & 1,40 & 3,10 \\
\hline Sloanea sp. & 15 & 0,59 & 0,3086 & 0,68 & 30 & 1,40 & 2,67 \\
\hline Ilex sp. & 10 & 0,39 & 0,5692 & 1,25 & 20 & 0,93 & 2,58 \\
\hline Myrtaceae 1 & 25 & 0,98 & 0,061 & 0,13 & 30 & 1,40 & 2,52 \\
\hline Blepharocalyx salicifolius & 30 & 1,18 & 0,1714 & 0,38 & 20 & 0,93 & 2,49 \\
\hline Vernonia discolor & 15 & 0,59 & 0,2119 & 0,47 & 30 & 1,40 & 2,46 \\
\hline Aegiphila sellowiana & 20 & 0,79 & 0,1051 & 0,23 & 30 & 1,40 & 2,42 \\
\hline Capsicodendron dinisii & 15 & 0,59 & 0,3869 & 0,85 & 20 & 0,93 & 2,38 \\
\hline Sapium glandulatum & 10 & 0,39 & 0,4727 & 1,04 & 20 & 0,93 & 2,37 \\
\hline Gochnatia polymorpha & 5 & 0,20 & 0,7468 & 1,64 & 10 & 0,47 & 2,31 \\
\hline N.i. 1 & 30 & 1,18 & 0,0528 & 0,12 & 20 & 0,93 & 2,23 \\
\hline Alchornea triplinervia & 15 & 0,59 & 0,1009 & 0,22 & 30 & 1,40 & 2,21 \\
\hline Lamanonia speciosa & 5 & 0,20 & 0,6724 & 1,48 & 10 & 0,47 & 2,14 \\
\hline Neomitranthes sp. & 15 & 0,59 & 0,0577 & 0,13 & 30 & 1,40 & 2,12 \\
\hline Piptocarpha sp. & 20 & 0,79 & 0,167 & 0,37 & 20 & 0,93 & 2,09 \\
\hline Myrsine ferruginea & 20 & 0,79 & 0,151 & 0,33 & 20 & 0,93 & 2,05 \\
\hline Picramnia parvifolia & 10 & 0,39 & 0,1339 & 0,29 & 20 & 0,93 & 1,62 \\
\hline Rollinia rugulosa & 10 & 0,39 & 0,1073 & 0,24 & 20 & 0,93 & 1,56 \\
\hline Tibouchina sellowiana & 10 & 0,39 & 0,0192 & 0,04 & 20 & 0,93 & 1,37 \\
\hline Lauraceae 1 & 5 & 0,20 & 0,2359 & 0,52 & 10 & 0,47 & 1,18 \\
\hline Ocotea c.f. catharinensis & 5 & 0,20 & 0,1894 & 0,42 & 10 & 0,47 & 1,08 \\
\hline Cinnamomum vesiculosum & 5 & 0,20 & 0,184 & 0,40 & 10 & 0,47 & 1,07 \\
\hline Ocotea sp. & 5 & 0,20 & 0,1733 & 0,38 & 10 & 0,47 & 1,04 \\
\hline Symplocos uniflora & 5 & 0,20 & 0,1579 & 0,35 & 10 & 0,47 & 1,01 \\
\hline Psychotria suterella & 10 & 0,39 & 0,0142 & 0,03 & 10 & 0,47 & 0,89 \\
\hline Ilex theezans & 10 & 0,39 & 0,0135 & 0,03 & 10 & 0,47 & 0,89 \\
\hline Trema micrantha & 5 & 0,20 & 0,0637 & 0,14 & 10 & 0,47 & 0,80 \\
\hline Roupala brasiliensis & 5 & 0,20 & 0,0545 & 0,12 & 10 & 0,47 & 0,78 \\
\hline Solanum sp.2 & 5 & 0,20 & 0,0279 & 0,06 & 10 & 0,47 & 0,73 \\
\hline Myrceugenia ovata & 5 & 0,20 & 0,0201 & 0,04 & 10 & 0,47 & 0,71 \\
\hline Soracea bonplandii & 5 & 0,20 & 0,0193 & 0.04 & 10 & 0,47 & 0,71 \\
\hline Coussarea sp. & 5 & 0,20 & 0,0175 & 0,04 & 10 & 0,47 & 0,70 \\
\hline Machaerium c.f. paraquariensis & 5 & 0,20 & 0,0144 & 0,03 & 10 & 0,47 & 0,70 \\
\hline Asteraceae 1 & 5 & 0,20 & 0,0102 & 0,02 & 10 & 0,47 & 0,69 \\
\hline Rubiaceae 2 & 5 & 0,20 & 0,0102 & 0,02 & 10 & 0,47 & 0,69 \\
\hline Eugenia cereja & 5 & 0,20 & 0,009 & 0,02 & 10 & 0,47 & 0,68 \\
\hline Myrcia selloi & 5 & 0,20 & 0,009 & 0,02 & 10 & 0,47 & 0,68 \\
\hline Campomanesia xanthocarpa & 5 & 0,20 & 0,009 & 0,02 & 10 & 0,47 & 0,68 \\
\hline Calyptranthes qrandifolia & 5 & 0,20 & 0,0078 & 0,02 & 10 & 0,47 & 0,68 \\
\hline Nectandra c.f. lanceolata & 5 & 0,20 & 0,0078 & 0,02 & 10 & 0,47 & 0,68 \\
\hline Ficus sp. & 5 & 0,20 & 0,0057 & 0,01 & 10 & 0,47 & 0,68 \\
\hline Rubiaceae 1 & 5 & 0,20 & 0,0057 & 0,01 & 10 & 0,47 & 0,68 \\
\hline Gomidesia affinis & 5 & 0,20 & 0,0053 & 0,01 & 10 & 0,47 & 0,68 \\
\hline Mortas & 170 & 6,68 & 2,5958 & 5,71 & 90 & 4,21 & 16,60 \\
\hline TOTAL & 2545 & 100,00 & 45,4611 & 100,00 & 2140 & 100,00 & 300,00 \\
\hline
\end{tabular}


Embora a Araucária seja a espécie mais importante nas duas áreas, marcando a fisionomia dos fragmentos, existem diferenças pronunciadas quanto ao grau de conservação. A presença de espécies pioneiras e secundárias iniciais no grupo das mais importantes evidencia que ambas as áreas estão em processo de avanço sucessional a fim de reconfigurar a estrutura típica da Floresta Om- brófila Mista. Como exemplos podem-se citar a Casearia decandra, a Myrsine umbellata e a Aegiphylla sellowiana, na área A e a Gordonia fruticosa, na área B. Considera-se que a área B está em melhor grau de conservação do que a área A, pela presença de espécies longevas e clímax entre as mais importantes, como a Ocotea odorifera, a Nectandra megapotamica e a Ilex paraguariensis.

\section{Figura 2 - Ordenamento estrutural da floresta na área A; onde DR Densidade Relativa, DoR} - Dominância Relativa, FR - Freqüência Relativa, PI - Porcentagem de Importância.

Figure 2 - Structural arrangement of the Forest on Area A; where DR Relative Density, DoR - Relative Dominance, Fr -Relative Frequency, PI - Importance Percentage.

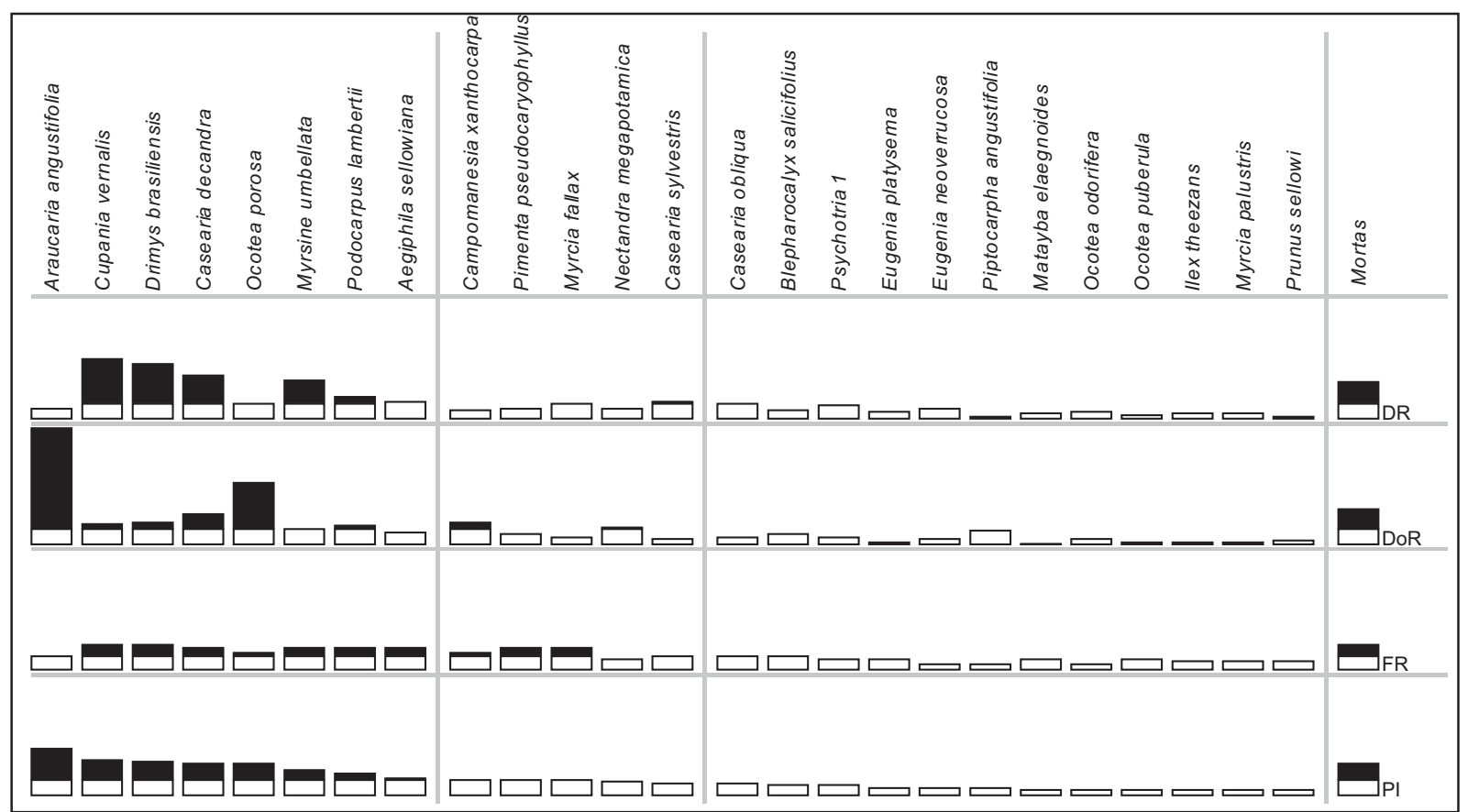




\section{Figura 3 - Ordenamento estrutural da floresta na área B; onde DR Densidade relativa, DoR - dominância relativa, FR - freqüência relativa, PI - porcentagem de importância.}

Figure 3 - Structural arrangement of the Forest on Area B; where DR Relative Density, DoR - Relative Dominanœ, Fr -Relative Frequency, PI - Importanœe Perœentage.

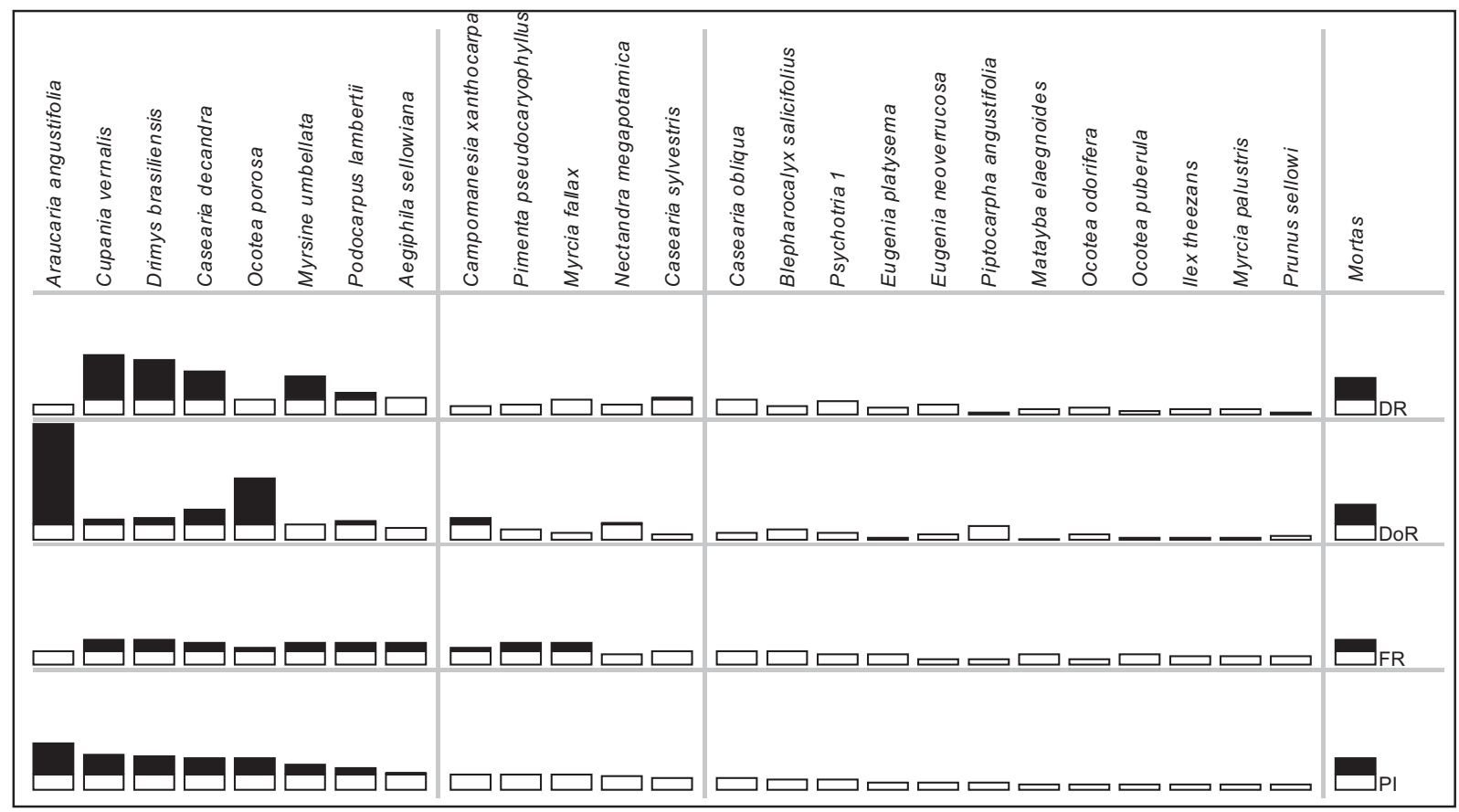

\section{Conclusões}

Ambos os remanescentes são caracterizados pela ocorrência da Araucaria angustifolia, espécie dominante e de grande valor de importância, acompanhada por diferentes espécies que determinam a fisionomia atual da floresta. Na área A merecem destaque Cupania vernalis, Drimys brasiliensis e Casearia decandra, com valores de importância acima da média. Na área B vale destacar a presença de Nectandra megapotamica, Ilex paraguariensis e Ocotea odorifera, espécies típicas de Floresta Ombrófila Mista, muito procuradas para exploração comercial e, dada sua importância estrutural da floresta, determina um maior grau de conservação desse ambiente.

Poucas espécies apresentam freqüência e densidade elevada; a maioria das espécies apresenta uma distribuição irregular, sendo que algumas delas ocorrem apenas em uma ou duas parcelas.

Claramente percebe-se que, em ambos os remanescentes, há um equilíbrio na distribuição das classes de diâmetro, onde há indivíduos para reposição quando morrem aqueles pertencentes às classes superiores, proporcionando uma continuidade à floresta pela rápida reocupação do espaço.

A estratificação demonstra que, provavelmente, a ausência de espécies de grande porte, na área A, é devido ao local ter sido submetido a um desbaste seletivo no passado. Já a área B não se mostrou definida e apresentou um número significativo de indivíduos distribuídos em todas as classes, demonstrando que a exploração madeireira neste local foi mínima.

Em termos do estudo de conservação, merece destaque a área $\mathrm{B}$, pois devido às alterações terem sido menos significativas, mantém características florísticas e estruturais próximas às da Floresta Ombrófila Mista original, bem como representa um remanescente de maior dimensão atenuando os prejuízos dinâmicos de desenvolvimento comum sem fragmentos florestais.

\section{Agradecimentos}

A todos que contribuíram direta ou indiretamente para a realização deste trabalho, espe- 
cialmente: ao Botânico Marcos Sobral, da Universidade Federal de Minas Gerais, pela identificação botânica de algumas espécies; ao colega Luiz Carlos de Oliveira Santos, pela colaboração na coleta de dados; ao Sr. Beno Bührer, pela permissão da realização do trabalho em sua Fazenda e ao Prof. Dr. Sylvio Péllico Netto, pela autorização da coleta de dados nas áreas do projeto PELD/CNPq.

\section{Referências}

GALVÃO, F; KUNIYOSHI, Y. S; RODERJAN, C. V. Levantamento fitossociológico das principais associações arbóreas da floresta nacional de Irati - PR. Floresta, Curitiba, v.19, n. 1/2, p. 30-49, 1989.

HUECK, K. As florestas da América do Sul. Brasília: Editora da Universidade de Brasília, 1972. p. 466.

IBGE. Manual técnico da vegetação brasileira. Rio de Janeiro, 1992. p. 92.

JARENKOW, J. A. Composição florística e estrutura da mata com araucária na Estação Ecológica de Aracuri, Esmeralda, Rio Grande do Sul. Dissertação (Mestrado) - Botânica, UFRS, Porto Alegre, 1985.

KLEIN, R.M. (a) Ecologia da flora e vegetação do Vale do Itajaí. Sellowia. Itajaí, 1979.

LONGHI, S. J. A Estrutura de uma floresta natural de Araucaria angustifolia (Bert.) O. Ktze, no Sul do Brasil. Dissertação (Mestrado em Engenharia Florestal) - UFPR, Curitiba, 1980.

MAACK, R. Geografia física do Estado do Paraná. Curitiba: CODEPAR, 1968.
MINISTÉRIO DO MEIO AMBIENTE. A floresta com araucária no Paraná: conservação e diagnóstico dos remanescentes florestais. Fundação de Pesquisas Florestais: Projeto de Conservação e Utilização Sustentável da Diversidade Biológica Brasileira - PROBIO; Brasilia: Ministério do Meio Ambiente, 2004. p. 236.

NASCIMENTO, A. R. T; LONGHI, S. J; BRENA, D. A. Estrutura e padrões de distribuição espacial de espécies arbóreas em uma amostra de Floresta Ombrófila Mista em Nova Prata, RS. Ciência Florestal, Santa Maria, v. 11, n. 1, p. 105-119, 2001.

RONDON NETO, R. M; WATZLAWICK, L F; CALDEIRA, M. V. W; SCHOENINGER, E. R. Análise florística e estrutural de um fragmento de Floresta Ombrófila Mista Montana, situado em Criúva, RsBrasil. Ciência Florestal, Santa Maria: v. 12, n. 1, p. 29-37, 2002.

SEMA/GTZ. Lista vermelha de plantas ameaçadas de ex tinção no estado do Paraná. Curitiba: Secretaria de Estado do Meio Ambiente, PR / GTZ, 1995.

SILVA, J. A; SALOMÃO, A. N; MARTINS NETTO, D. A. Estrutura, fitossociologia e regeneração Natural da Reserva Genética de Caçador - S.C. In: CONGRESSO FLORESTAL PANAMERICANO, 1., CONGRESSO FLORESTALBRASILEIRO , 7; Curitiba, 1993 Anais... Curitiba: SBS/ SBEF, 1993. v. 1, p. 347-352.

Recebido: 03/09/2004

Aprovado: 31/01/2005 